\title{
Superoxide dismutase 3 protects mesenchymal stem cells through enhanced autophagy and regulation of FoxO3a trafficking
}

\author{
Gaurav Agrahari ${ }^{\#}$, Shyam Kishor Sah ${ }^{\#}$ E Tae-Yoon Kim ${ }^{*}$ \\ Laboratory of Dermato-Immunology, College of Medicine, The Catholic University of Korea, Seoul 06591, Korea
}

\begin{abstract}
Therapeutic applications of mesenchymal stem cells (MSCs) are limited due to their early death within the first few days of transplantation. Therefore, to improve the efficacy of cellbased therapies, it is necessary to manipulate MSCs so that they can resist various stresses imposed by the microenvironment. Moreover, the role of superoxide dismutase 3 (SOD3) in regulating such survival under different stress conditions remain elusive. In this study, we overexpressed SOD3 in MSCs (SOD3-MSCs) and evaluated its effect under serum starvation conditions. Nutritional limitation can decrease the survival rate of transplanted MSCs and thus can reduce their efficacy during therapy. Interestingly, we found that SOD3-MSCs exhibited reduced reactive oxygen species levels and greater survival rates than normal MSCs under serum-deprived conditions. In addition, overexpression of SOD3 attenuated starvationinduced apoptosis with increased autophagy in MSCs. Moreover, we have demonstrated that SOD3 protects MSCs against the negative effects of serum deprivation via modulation of AMP-activated protein kinase/sirtulin 1, extracellular signalregulated kinase activation, and promoted Forkhead box O3a trafficking to the nucleus. Taken together, these results demonstrate that SOD3 promotes MSCs survival and add further evidence to the concept that SOD3-MSCs may be a potential therapeutic agent with better outcomes than normal MSCs for various diseases involving oxidative stress and compromised MSCs survival during therapy. [BMB Reports 2018; 51(7): 344-349]
\end{abstract}

*Corresponding author. Tel: +82-2-2258-7630; Fax: +82-2-34828261; E-mail: tykimder@catholic.ac.kr

${ }^{\#}$ These authors contributed equally to this work.

https://doi.org/10.5483/BMBRep.2018.51.7.078

Received 11 April 2018, Revised 27 April 2018, Accepted 24 May 2018

Keywords: Apoptosis, Autophagy, Mesenchymal stem cell, Serum starvation, Superoxide dismutase 3

\section{INTRODUCTION}

Mesenchymal stem cells (MSCs) can be used in cell-based therapies to treat several diseases such as tissue injuries, inflammatory diseases, cardiovascular diseases, and several skin diseases, because of their self-renewal, differentiation, immunomodulation, and regeneration capacities (1-7). However, their poor survival during such therapy is a major obstacle in their implementation. The therapeutic implications of MSCs are laid down by various undesirable factors such as nutrient deprivation, oxidative stress, inflammation, and many others, resulting in low cell viability (8-11). Prolonged nutrient starvation is one of the major problems faced by engrafted MSCs $(12,13)$, which indicates that increasing the survival of MSCs can enhance the efficacy of MSC-based therapies.

Autophagy is a conserved intracellular mechanism for maintaining cellular homeostasis, particularly during starvation or stress $(14,15)$. Various studies have elucidated important role of autophagy in MSCs. Autophagy is essential to maintaining the cellular stemness and differentiation of MSCs (16). Similarly, autophagy in MSCs improves their wound healing capacity (17) and immunosuppressive properties (18). In addition, autophagy can degrade aggregate-prone intracytoplasmic proteins which are responsible for various neurodegenerative diseases, and it can also protect against certain infectious diseases (19). Therefore, the medical importance of autophagy as well as its therapeutic potential still need to be addressed.

Superoxide dismutase (SOD) is an antioxidant enzyme that detoxifies the superoxide radical and exists in three isoforms in mammals: SOD1 (Cu, Zn-SOD, cytosol and nucleus), SOD2 (Mn-SOD, mitochondria), and SOD3 (Cu, Zn-SOD, extracellular matrix). Though they catalyze similar reactions, SOD3 is preferred because of its longer half-life (20-24 hours) and immunomodulatory effect during the inflammatory response. SOD3 has wide clinical applications due to its anti-chemotactic, anti-inflammatory, and anti-tumorigenic activity. The therapeutic implications of SOD3 in various skin diseases such as psoriasis and atopic dermatitis have been well-studied. It has previously been shown that treatment with antioxidants can enhance the survival and therapeutic efficacy of MSCs $(20,21,22)$.

ISSN: 1976-670X (electronic edition)

Copyright (C) 2018 by the The Korean Society for Biochemistry and Molecular Biology

(c) This is an open-access article distributed under the terms of the Creative Commons Attribution Non-Commercial License (http://creativecommons.org/licenses/by-nc/4.0) which permits unrestricted non-commercial use, distribution, and reproduction in any medium, provided the original work is properly cited. 
Similarly, MSCs were found to promote cerebellar neuronal survival through the secretion of SOD3 (23). However, the mechanism of SOD3 on this regulation of cell survival needs to be further investigated.

In this study, we utilized serum deprivation conditions to imitate nutritional starvation in MSCs and investigated the effect of SOD3 on MSC survival. Here, we showed that MSCs can survive longer under stress conditions through the increased secretion of SOD3, and defined the intracellular mechanism underlying this enhanced MSC survival through the overexpression of SOD3. Our findings suggest that transplantation of SOD3-transduced MSCs may serve as an effective therapy for several disorders that involve oxidative stress.

\section{RESULTS}

\section{SOD3 offers significant survival potential to MSCs under serum starvation conditions}

To investigate the protective effects of SOD3 in MSCs under serum deprivation-mediated cytotoxicity, MSCs or SOD3-MSCs were cultured in serum-free medium for five days, and superoxide anion productions as well as cell survival were both evaluated. Our data showed that SOD3-MSCs exhibited reduced superoxide anion production (Fig. 1A) and significantly enhanced cell survival compared to MSCs under serum deprivation conditions, as evaluated through microscopic

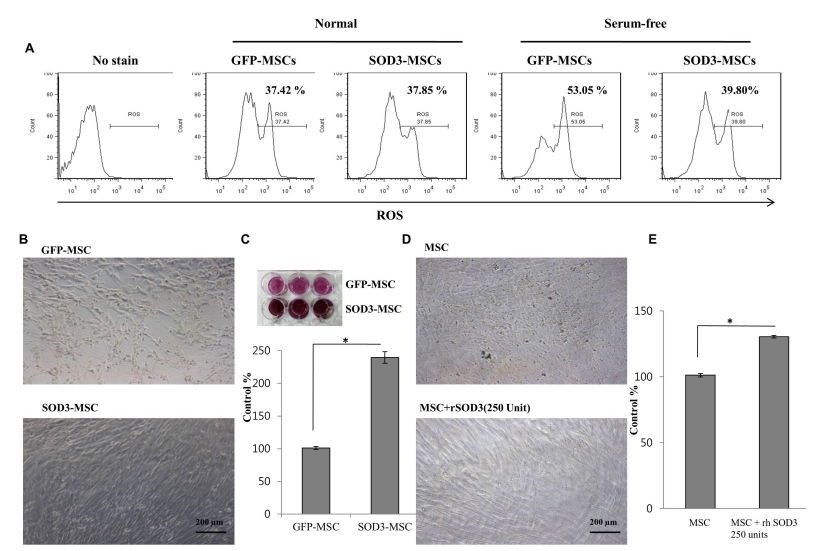

Fig. 1. SOD3 overexpression in MSCs reduced ROS and increased survival under serum starvation. (A) ROS production in SOD3-transduced MSCs and MSCs under serum starvation was assessed by staining with DHE $(10 \mu \mathrm{M})$, and FACS analysis followed. (B, C) SOD3-MSCs and normal MSCs were exposed to chronic serum starvation for five days, after which the viability was determined through microscopic examination at $100 \times$ magnification and MTT assay. (D, E) MSCs were treated with human recombinant SOD3 and viability was measured by microscopic examination at $100 \times$ magnification and MTT assay. Experiments were performed in triplicate. Data are expressed as mean \pm standard deviation. ${ }^{*} P<0.05$ level. examination (Fig. 1B) and indicated by a higher reduction of tetrazolium dye to purple formazen during MTT assay (Fig. 1C). Treatment with human recombinant SOD3 alone recapitulated the enhanced survival effect in MSCs under serum-free conditions as analyzed through both microscopic examination and MTT assay (Fig. 1D, E).

\section{SOD3 inhibited starvation-induced apoptosis, promoted} double-strand break repair protein expression in MSCs

To further evaluate the role of SOD3 in starvation-induced cell death, apoptosis was analyzed using caspase-3 by western blot. SOD3 markedly showed reduced apoptosis in contrast to normal MSCs, as indicated by a reduced caspase-3 level (Fig. 2A). Next, we evaluated the mRNA expression of different anti-apoptotic and pro-apoptotic genes as well as of several regulatory genes that participate in cell survival, cell damagerepair, and maintaining the stemness of MSCs. Overexpression of SOD3 in MSCs exhibited no significant effects on anti-apoptotic molecules like BCL-2 and BCL-XI, but markedly reduced the expression of pro-apoptotic molecules such as Bax at mRNA levels under serum starvation conditions (Fig. 2B). In contrast, overexpression of SOD3 in MSCs did not affect cell cycle regulators such as CDK2, CDK4, and Cyclin E1, cell senescence factors like p16 and RB2, stemness-related transcription factors like Oct-4, klf4, and Nanog, or ROS modulator ROMO1 at mRNA levels. Interestingly, a sharp reduction in the expression of $\mathrm{P} 21$ by SOD3 indicated that

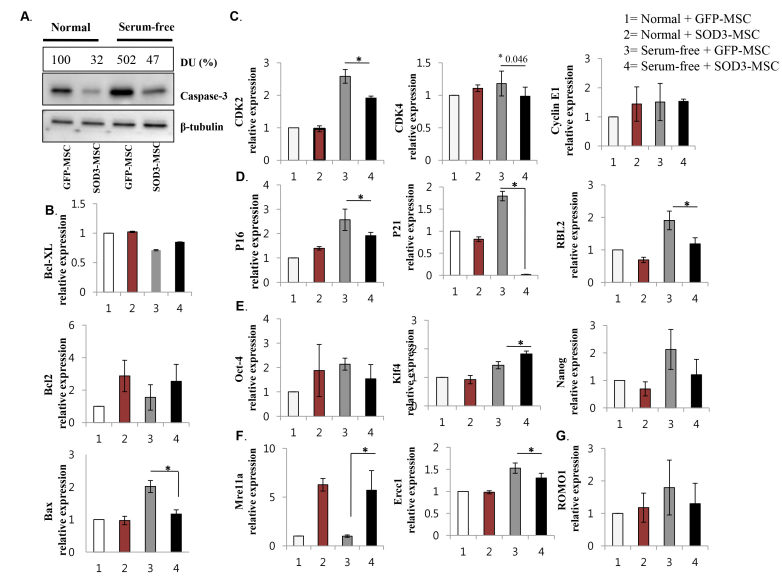

Fig. 2. SOD3 overexpression in MSCs showed reduced apoptosis and had no significant effects on the genes involved in different cellular processes under serum-starved conditions. (A) Expression of Caspase-3 was analyzed through western blotting. The numerical values on the blot represent \% relative densitometric unit (DU), as measured by Image software. (B-G) RNA expression of genes involved in apoptosis, cell cycling, cell senescence, stemness, DNA repair, and ROS modulator under serum-starved conditions were analyzed through qRT-PCR. Experiments were performed in triplicate. Data are expressed as mean \pm standard deviation. ${ }^{*} \mathrm{P}<0.05$ level. 
SOD3 may not allow the cells to undergo growth arrest (Fig. 2D, second panel). Moreover, SOD3 induced the expression of double-strand DNA repair protein Mre11a at mRNA levels under serum-free conditions, suggesting that SOD3 can help overcome any damage to the DNA of the cells which are required for cell survival and growth (Fig. 2F, first panel).

\section{SOD3 promoted autophagy by upregulating SIRT1 and P-AMPK}

Autophagy is induced in serum-deprived conditions in order to provide cells with additional internal nutrient supplies. Therefore, we hypothesize that SOD3 may regulate autophagy and promote cell survival under serum-free conditions as well. Here, we showed that the starvation condition indeed induced the autophagy which is essential for transient cell survival, but overexpression of SOD3 in MSCs under serum-free conditions also significantly enhanced the conversion of LC3-II level at 48 hours. During nutrient starvation, autophagy is brought on through the activation of either of two energy sensors: AMP-activated protein kinase (AMPK) (24) or sirtulin1 (SIRT1) (25). Thus, we next evaluated whether SOD3 had any effect on the activation of AMPK and SIRT1. Our data showed that overexpression of SOD3 in MSCs significantly promoted the activation of AMPK and SIRT1 at both 6 and 48 hours compared to MSCs alone under serum-free conditions (Fig. 3A). However, a key regulator of autophagy initiation and progression, molecule Unc-51-like autophagy activating kinase (ULK1), was not affected at either time point (Fig. 3B).

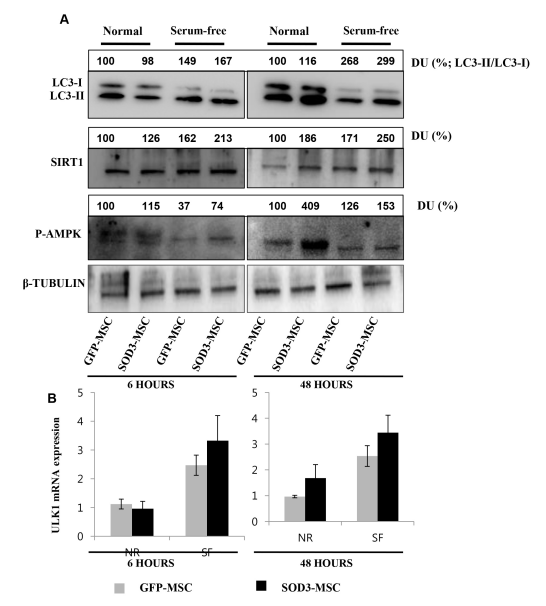

Fig. 3. Increased autophagy induction and upregulation of SIRT1 and P-AMPK expression in SOD3-MSCs. (A) Expressions of LC3, SIRT1, and P-AMPK were analyzed through western blotting. (B) RNA expression for ULK 1 was analyzed through qRT-PCR. The numerical values on the blot represent $\%$ relative densitometric unit (DU), as measured by Imagej software. Control groups were set as $100 \%$ DU in each regimen. Experiments were performed in triplicate. Data are expressed as mean \pm standard deviation. $* P<0.05$ level.

\section{SOD3 inhibited Akt activation and promoted the translocation of FoxO3a into the nucleus}

A variety of tyrosine kinase receptors, including the insulin receptor, activate the PI3K pathway, which leads to the activation of both mammalian target of rapamycin complex 1 (TORC1) and mammalian target of rapamycin complex 2 (mTOCR2) under calorie restriction or other stress conditions (26). mTORC2 can phosphorylate and activate Akt. Activated Akt can phosphorylate Forkhead box $\mathrm{O}$ (FoxO) transcription factors, restricting their localization to the cytosol. FoxOs that translocate to the nucleus can transcriptionally activate the expression of a variety of genes, including protein-folding chaperones, antioxidant enzymes, and metabolic regulators. Activated Akt can also activate mTORC1, and mTORC1 activates the mechanisms that promote protein translation and lipid and nucleic acid synthesis and inhibit autophagy (27). Interestingly, we found that SOD3 inhibits Akt activation in MSCs and promotes the timely translocation of FoxO3a into the nucleus under chronic serum deprivation conditions, suggesting that SOD3 may increase MSC survival by facilitating reactive oxygen species (ROS) removal and promoting other beneficial cellular mechanisms, which confers resistance to oxidative stress under serum deprivation (Fig. 4A, B). Furthermore, it has also been shown that the extracellular signal-regulated kinase (ERK) pathway can play a positive role in autophagy induction and cell survival (28).

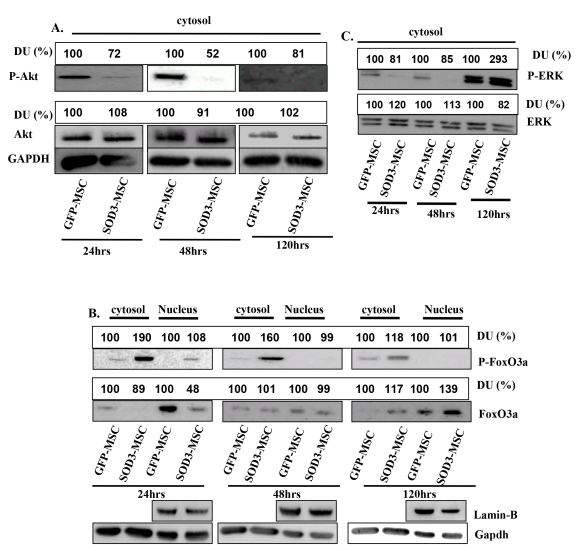

Fig. 4. SOD 3 inhibited Akt signaling in MSCs and promoted the translocation of FoxO3a into the nucleus under starvation conditions. (A) MSCs were overexpressed with SOD3 and GFP and then subjected to serum starvation. Phosphorylated Akt at serine 473 and total Akt were analyzed through western blotting. (B) Translocation of FoxO3a into the nucleus was determined by isolating cytosolic and nuclear protein and analyzed through western blotting. (C) Total Erk and its phosphorylated form were determined through western blotting. The numerical values on the blot represent \% relative densitometric unit (DU), as measured by Image software. Control groups were set as 100\% DU in each regimen. Experiments were performed in triplicate. Data are expressed as mean \pm standard deviation. 
Overexpression of SOD3 promoted the activation of ERK at 120 hours of chronic starvation significantly more than normal MSCs (Fig. 4C).

\section{DISCUSSION}

In this study, we first determined through flow cytometry that overexpression of SOD3 in MSCs does not significantly affect the cell size, complexity, and stemness of MSCs under normal, serum-free, or glucose-serum-free conditions (Supplementary Fig. 1). However, the glucose-serum-free condition was removed from this experiment due to an excessive death of cells. Furthermore, we showed that overexpression of SOD3 in MSCs increased their survival under serum deprivation conditions.

Serum deprivation induces apoptosis and leads to cell death (29). The reduction in caspase- 3 and pro-apoptotic genes in SOD3-MSCs suggests that SOD3 can inhibit cells undergoing apoptosis during starvation condition, and in turn increase their chances of survival. Serum deprivation can affect many genes involved in cell cycle, cell senescence, stemness, and DNA repair, which play a major role in the survival of cells under stress conditions. The overexpression of SOD3 showed no significant effect on the genes CDK2, CDK4, Cyclin E1, P16, RBL2, OCT4, KLF4, Nanog, Ercc1, and Romo1. However, the upregulation of DNA repair gene MRE11A and reduction of P21, which is responsible for arresting cell growth, may be the reason for the promotion of the survival of cells under serum deprivation conditions in SOD3-MSCs.

Autophagy is the catabolic process that provides the cell with the essential constituents required for maintaining the metabolism for survival purposes under stress conditions. Here, we found that the level of autophagy increased starting from 3 hours and declined after 48 hours. This reduction of autophagy may limit cell survival under starvation conditions. Similarly, a positive correlation was found between the endogenous level of SOD3 and autophagy induction as the level of SOD3 and LC3-II increased starting from 3 hours and declined after 48 hours (Supplementary Fig. 2), suggesting that SOD3 may be a factor in the regulation of autophagy. Taking this into consideration, we examined the level of autophagy in SOD3-MSCs and found that SOD3-MSCs showed a higher level of autophagy induction than normal MSCs. Similarly, the activation of the two energy sensors of the cells, AMPK and SIRT1, positively regulates autophagy during starvation (24, 25). Our results showed a higher level of these two sensing elements in SOD3-MSCs than in normal MSCs. However, further investigation is required to properly elucidate this relationship.

Protein kinase B (PKB), also known as Akt, suppresses autophagy either through activation of mTOR, which in turn inhibits the autophagy-initiating ULK 1 kinase complex, or through the phosphorylation of Beclin 1 (30). Here, SOD3-MSCs suppressed the levels of phosphorylated Akt, suggesting that
SOD3 may induce autophagy through inhibiting the Akt signaling pathway. However, the effects of SOD3 on the downstream pathway of Akt are yet to be studied. FoxO3a has been found to be translocated into the nucleus, where they induce the expression of various autophagy genes such as LC3B, Atg12, Atg4B, beclin1, ulk2, Gabarapl1, vps 34, Bnip3l, and Bnip3 through directly binding to their promoters (31-36). Our results showed that SOD3-MSCs promote the translocation of FoxO3a into the nucleus. ERK is one of the conserved pathways involved in many biological processes such as cell proliferation, differentiation, apoptosis, and cell survival. Recent studies have shown that ERK plays an important role in the up-regulation of starvation-induced autophagy, either through the down-regulation of $\mathrm{Akt} / \mathrm{mTOR} / \mathrm{S} 6 \mathrm{~K}$ (37) or through the regulation of Beclin 1 (28). Activation of P-ERK in SOD3-MSCs further supports our hypothesis that SOD3 induces autophagy.

In summary, we can speculate that increases in the DNA repair gene MRE11A and decreases in the growth suppressor gene P21, down-regulation of ROS, apoptosis, and enhancement of autophagy may be the mechanisms of SOD3 for increasing the survival of cells under starvation conditions. Thus, SOD3 can be a promising candidate to promote cell survival and enhance the efficacy of MSCs therapy. However, the effects on survival of SOD3-MSCs need further confirmation in vivo in the future.

\section{MATERIALS AND METHODS}

\section{Cell culture and treatment}

Human umbilical cord blood-derived MSCs were isolated as previously described (38-40). MSCs were cultured in KSB-3 Basal medium (B1001, Irvine Scientific, USA) with $10 \%$ fetal bovine serum, $2.2 \mathrm{~g} / \mathrm{L}$ sodium bicarbonate, $100 \mathrm{U} / \mathrm{ml}$ penicillin, and $100 \mu \mathrm{g} / \mathrm{ml}$ streptomycin at $37^{\circ} \mathrm{C}$ in a humidified incubator with $5 \% \mathrm{CO}_{2}$. All experiments were carried out on MSCs from $6^{\text {th }}$ to $8^{\text {th }}$ passages. MSCs were then transduced with lentivirus vector expressing either human SOD3 or GFP (control) at a multiplicity of infection of 10 . The expression and activity of SOD3 can be found in the supplementary section (Supplementary Fig. 3). Serum deprivation conditions were imposed on the cells by using serum-free KSB-3 Basal medium.

\section{Cell survival assay}

MSC survival was evaluated through 3-(4,5-dimethylthiazol2-yl)-2,5-diphenyltetrazolium bromide (MTT) assay. Cells were seeded in 24 well plates at a density of $1 \times 10^{5}$ cells/well. After exposure to different stress conditions, $60 \mu \mathrm{l}$ of MTT reagent $(5 \mathrm{mg} / \mathrm{ml}$ in $1 \times$ PBS $)$ was added to each well and incubated at $37^{\circ} \mathrm{C}$ for 3-4 hours, followed by removal of medium and dissolution of cells in $500 \mu \mathrm{l}$ of Dimethyl sulfoxide (DMSO) per well. The absorbance was then measured at $595 \mathrm{~nm}$ by the microplate reader (Power wave XS2, Bio Tek, USA). 


\section{Quantitative real-time PCR (qRT-PCR) analysis}

Total RNA was isolated using trizol reagent (Life Technologies, Invitrogen). Complementary DNA was synthesized from $1 \mu \mathrm{g}$ of total RNA using the PrimeScript ${ }^{\mathrm{TM}}$ RT reagent Kit (RR047A,Clontech Takara Bio INC, Japan), and qRT-PCR was performed using the KAPA SYBR ${ }^{\mathbb{R}}$ FAST qPCRMaster Mix (2X) universal Kit (KAPA Biosystems, Cape town, South Africa) in a Rotor-Gene 6000 instrument (Corbett Life Science, Mortlake, NSW, Australia). The relative amount of mRNA was calculated using the $2^{-\Delta \Delta \mathrm{Ct}}$ method with glyceraldehyde 3-phosphate dehydrogenase (GAPDH) as an endogenous control. The primers used as well as their sequences can be found in the supplementary section (Supplementary Table 1).

\section{Western blot analysis}

MSCs were harvested and lysed in ice-cold radioimmunoprecipitation (RIPA) buffer (89901, Thermo Scientific, USA) containing protease inhibitor cocktail (Roche Diagnostic, Germany) for 30 minutes at $4^{\circ} \mathrm{C}$. An equal amount of proteins were loaded per lane and the blotted membranes were incubated overnight at $4^{\circ} \mathrm{C}$ with Caspase-3 (9662S, cell signaling), LC3A/B (4108S, cell signaling), phospo-AMPK $\alpha$ (Thr 172) (2535S, cell signaling), P-FoxO3a (9465S, cell signaling), FoxO3a (9467S, cell signaling), SIRT1 (H-300) (SC-15404, Santa cruz), P-Akt (SC-7985, Santa cruz), Akt (SC-8312, Santa cruz), $\beta$-tubulin (SC-9104, Santa cruz), SOD3 (ab 21974), GAPDH (SC-25778, Santa cruz), and Lamin-B (SC-6216, Santa cruz) antibodies (1:1000 dilution) followed by incubation for two hours with horseradish peroxidaseconjugated secondary antibody (1:5000 dilution). The blots were then detected with a western blot detection kit (WesternBright ${ }^{\mathrm{TM}} \mathrm{ECL}, \mathrm{USA}$ )

\section{Flow cytometry}

For Reactive oxygen species (ROS) measurement, MSCs were stained with dihydroethidium (DHE) (D1168, Life Technologies Corporation, Eugene, USA) at $10 \mu \mathrm{M}$ concentration for 15 minutes and then analyzed by BD FACSCanto". Cell size and cell complexity were analyzed by measuring forward and side scatter light, respectively. Data was analyzed with Flowjo software.

\section{Statistical analysis}

Each experiment was performed at least three times and all data were presented as mean \pm SD. We used Student's $t$-tests to determine significant differences wherever appropriate. Values of $\mathrm{P}<0.05$ were considered statistically significant.

\section{ACKNOWLEDGEMENTS}

This research was supported by the Bio and Medical Technology Development Program of the National Research Foundation (NRF) funded by Korean government (MSIT) (No. 2016M3A9B6903020) and by a grant of the Korea Health
Technology R\&D project through the Korea Health Industry Development Institute (KHIDI), funded by the Ministry of Health and Welfare, Republic of Korea (Grant No. HI17C0616).

\section{CONFLICTS OF INTEREST}

The authors have no conflicting interests.

\section{REFERENCES}

1. Biffi A, Montini E, Lorioli L et al (2013) Lentiviral hematopoietic stem cell gene therapy benefits metachromatic leukodystrophy. Science 341, 1233158

2. Garbern JC and Lee RT (2013) Cardiac stem cell therapy and the promise of heart regeneration cell. Stem Cell 12, 689-698

3. Greco SJ and Rameshwar P (2012) Mesenchymal stem cells in drug/gene delivery: implications for cell therapy. TherDeliv 3, 997-1004

4. Law S and Chaudhuri S (2013) Mesenchymal stem cell and regenerative medicine: regeneration versus immunomodulatory challenges. Am J Stem Cells 2, 22-38

5. Murphy MB, Moncivais K and Caplan Al (2013) Mesenchymal stem cells: environmentally responsive therapeutics for regenerative medicine. Exp Mol Med 45, 54

6. Przybyla LM, Theunissen TW, Jaenisch R and Voldman J (2013) Matrix remodeling maintains embryonic stem cell self-renewal by activating stat3. Stem Cells 31, 1097-1106

7. Saunders A, Faiola F and Wang J (2013) Concise review: pursuing self-renewal and pluripotency with the stem cell factor Nanog. Stem Cells 31, 1227-1236

8. Haider $\mathrm{H}$ and Ashraf $\mathrm{M}$ (2010) Preconditioning and stem cell survival. J Cardiovasc Transl Res 3, 89-102

9. Zhou H, Yang J, Xin T et al (2014) Exendin-4 protects adipose-derived mesenchymal stem cells from apoptosis induced by hydrogen peroxide through the PI3K/AktSfrp2 pathways. Free Radic Biol Med 77, 363-375

10. Li CJ, Sun LY and Pang CY (2015) Synergistic protection of $\mathrm{N}$-acetylcysteine and ascorbic acid 2-phosphate on human mesenchymal stem cells against mitoptosis, necroptosis and apoptosis. Sci Rep 5, 9819

11. Zhang Q, Liu S, Li T et al (2016) Preconditioning of bone marrow mesenchymal stem cells with hydrogen sulfide improves their therapeutic potential. Oncotarget 7, 58089-58104

12. Zhu W, Chen J, Cong X, Hu S and Chen X (2006) Hypoxia and serum deprivation-induced apoptosis in mesenchymal stem cells. Stem Cells 24, 416-425

13. Potier E, Ferreira E, Meunier A, Sedel L, LogeartAvramoglou D and Petite H (2007) Prolonged hypoxia concomitant with serum deprivation induces massive human mesenchymal stem cell death. Tissue Eng 13, 1325-1331

14. Mizushima N and Klionsky DJ (2007) Protein turnover via autophagy: implications for metabolism. Annu Rev Nutr 27, $19-40$

15. Stolz A, Ernst A and Dikic I (2014) Cargo recognition and 
trafficking in selective autophagy. Nat Cell Biol 16, 495-501

16. Sbrana FV, Cortini M, Avnet S, Perut F, Columbaro M, De Milito A and Baldini N (2016) The Role of Autophagy in the Maintenance of Stemness and Differentiation of Mesenchymal Stem Cells. Stem Cell Rev 12, 621-633

17. An Y, Liu WJ, Xue P et al (2018) Autophagy promotes MSC-mediated vascularization in cutaneous wound healing via regulation of VEGF secretion. Cell Death Dis 9,58

18. Gao L, Cen S, Wang P et al (2018) Autophagy Improves the Immunosuppression of $\mathrm{CD}^{+} \mathrm{T}$ Cells by Mesenchymal Stem Cells Through Transforming Growth Factor- $\beta 1$. Stem Cells Transl Med 5, 1496-1505

19. Rubinsztein DC, Bento CF and Deretic V (2015) Therapeutic targeting of autophagy in neurodegenerative and infectious diseases. J Exp Med 212, 979-990

20. Zeng W, Xiao J, Zheng G et al (2015) Antioxidant treatment enhances human mesenchymal stem cell anti-stress ability and therapeutic efficacy in an acute liver failure model. Sci Rep 5, 11100

21. Kim PH, Na SS, Lee B, Kim JH and Cho JY (2015) Stanninocalcin 2 enhances mesenchymal stem cell survival by suppressing oxidaative stress. BMB Rep 48, 702-707

22. Suh N and Lee Eb (2017) Antioxidant effects of selenocysteine on replicative senescence in human adipose-derived mesenchymal stem cells. BMB Rep 50, 572-577

23. Kemp K, Hares K, Mallam E, Heesom KJ, Scolding N and Wilkins A (2010) Mesenchymal stem cell-secreted superoxide dismutase promotes cerebellar neuronal survival. J Neurochem 114, 1569-1580

24. Egan DF, Shackelford DB, Mihaylova MM et al (2011) Phosphorylation of ULK1 (hATG1) by AMP-activated protein kinase connects energy sensing to mitophagy. Science 331, 456-461

25. Kim J, Kundu M, Viollet B and Guan KL (2011) AMPK and mTOR regulate autophagy through direct phosphorylation of Ulk1. Nat Cell Biol 13, 132-141

26. Laplante M and Sabatini DM (2012) mTOR signaling in growth control and disease. Cell 149, 274-293

27. Salih DA and Brunet A (2008) FoxO transcription factors in the maintenance of cellular homeostasis during aging. Curr Opin Cell Biol 20, 126-136

28. Wang j, Whiteman MW, Lian $\mathrm{H}$ et al (2009) A Non-canonical MEK/ERK Signaling Pathway Regulates Autophagy via Regulating Beclin 1. J Biol Chem 284,
$21412-21424$

29. Lee SB, Kim JJ, Kim TW, Kim BS, Lee MS and Yoo YD (2010) Serum deprivation induced reactive oxygen species production is mediated by Romo1. Apoptosis 15, 204-218

30. Richard CW, Yongjie W, Zhenyi A et al (2012) Akt-Mediated Regulation of Autophagy and Tumorigenesis Through Beclin 1 Phosphorylation. Science 338, 956-959

31. Mammucari C, Milan G, Romanello V et al (2007) FoxO3 controls autophagy in skeletal muscle in vivo. Cell Metab $6,458-471$

32. Mammucari C, Schiaffino S and Sandri M (2008) Downstream of Akt: FoxO3 and mTOR in the regulation of autophagy in skeletal muscle. Autophagy 4, 524-526

33. Van der Vos KE, Eliasson $P$, Proikas-Cezanne $T$ et al (2012) Modulation of glutamine metabolism by the $\mathrm{PI}(3) \mathrm{K}-\mathrm{PKB}-\mathrm{FOXO}$ network regulates autophagy. Nat Cell Biol 14, 829-837

34. Xiong X, Tao R, Depinho R and Charlie Dong X (2012) The autophagy-related gene 14 (Atg14) is regulated by Forkhead box $\mathrm{O}$ transcription factors and circadian rhythms and plays a critical role in hepatic autophagy and lipid metabolism. J Biol Chem 287, 39107-39114

35. Xu P, Das M, Reilly J and Davis RJ (2011) JNK regulates FoxO-dependent autophagy in neurons. Genes Dev 25, 310-322

36. Kume S, Uzu T, Horiike K et al (2010) Calorie restriction enhances cell adaptation to hypoxia through Sirt1dependent mitochondrial autophagy in mouse aged kidney. J Clin Invest 120, 1043-1055

37. Shinojima N, Yokoyama T, Kondo Y and Kondo S (2007) Roles of the Akt/mTOR/p70S6K and ERK1/2 signaling pathways in curcumin-induced autophagy. Autophagy 3, 635-637

38. Divya MS, Roshin GE, Divya TS et al (2012) Umbilical cord blood-derived mesenchymal stem cells consist of a unique population of progenitors co-expressing mesenchymal stem cell and neuronal markers capable of instantaneous neuronal differentiation. Stem Cell Res Ther 3, 57

39. Reddy NP, Vemuri MC and Pallu R (2007) Isolation of stem cells from human umbilical cord blood. Methods Mol Biol 407, 149-163

40. Wang M, Yang Y, Yang D et at (2009) The immunomodulatory activity of human umbilical cord bloodderived mesenchymal stem cells in vitro. Immunology $126,220-232$ 